\title{
Digital Image Watermarking using MSLDIP (Modified Substitute Last Digit in Pixel)
}

\author{
Abdelmgeid A. Ali \\ Computer Science Dept. \\ Faculty of Science, \\ Minia University \\ Egypt
}

\author{
Ahmed A. Radwan \\ Computer Science Dept. \\ Faculty of Science, \\ Minia University \\ Egypt
}

\author{
Ahmed H. Ismail \\ Computer Science Dept. \\ Faculty of Science, \\ Minia University \\ Egypt
}

\begin{abstract}
The improvements in Internet technologies and growing requests on online multimedia businesses have made digital copyrighting as a significant challenge for businesses that are related with online content distribution via diverse business models including pay-per-view, subscription, trading, etc. Copyright protection and the proof for rightful ownership are major issues related with the distribution of any digital images. Digital watermarking is one of the suggested solutions for copyright protection of multimedia data. This technique is better than Digital Signatures and other methods because it does not rise overhead. In this paper a new watermarking method based on spatial domain image steganography method called MSLDIP (Modified Substitute Last Digit in Pixel) is proposed. The main goal of this method is to hide the watermark in the pixels of digital image in such a manner that the human visual system is not able to distinguish between the cover image and the watermarked image. Also the experimental results showed that this method can be used effectively in the field of watermarking.
\end{abstract}

\section{General Terms}

MSE (Mean Square Error), PSNR (Peak Signal to Noise Ratio).

\section{Keywords}

Watermarking, SLDIP (Substitute Last Digit in Pixel), MSLDIP (Modified Substitute Last Digit in Pixel), Spatial Domain.

\section{INTRODUCTION}

The term 'digital watermarking' was first time appeared in 1993, when Tirkel presented two watermarking methods to hide the watermark data in the cover images [1]. The success of the Internet, popular digital recording and cost effective and storage devices, and the promise of higher bandwidth and quality of service for each wired and wireless networks have made it possible to create, replicate, transmit, and distribute digital content in an easy way. The protection and implementation of intellectual property rights for digital media has become a significant issue [2]. Digital watermarking is that technology that provides and ensures security, copyright protection and data authentication to the digital content. Digital Image Watermarking is a technique to secure digital contents by integrating the original digital image and a watermark data [3]. Digital Watermarking techniques deduced from steganography, which means covered writing (from the Greek words stegano that means "covered" and graphos that means "to write"). Steganography is the science of communicating information while hiding the existence of the communication [4]. Digital watermarking techniques can be classified into two main categories on the basis of watermark embedding domain, i.e. spatial domain (which converges on modifying the pixels of one or two randomly selected subsets of images) and frequency domain (which values of certain frequencies are modified from their original) [5].

\section{BASIC WATERMARKING SYSTEM}

Digital Watermarking System mostly consists of two basic parts [3] (Figure 2.1):

i. Watermark Embedder: is responsible for embedding the watermark image and watermark key into the cover image (original).

ii. Watermark Detector: is responsible for finding and extracting the watermark image with the only using watermark key and watermarked image. This part executes on the other side after transmission of data.

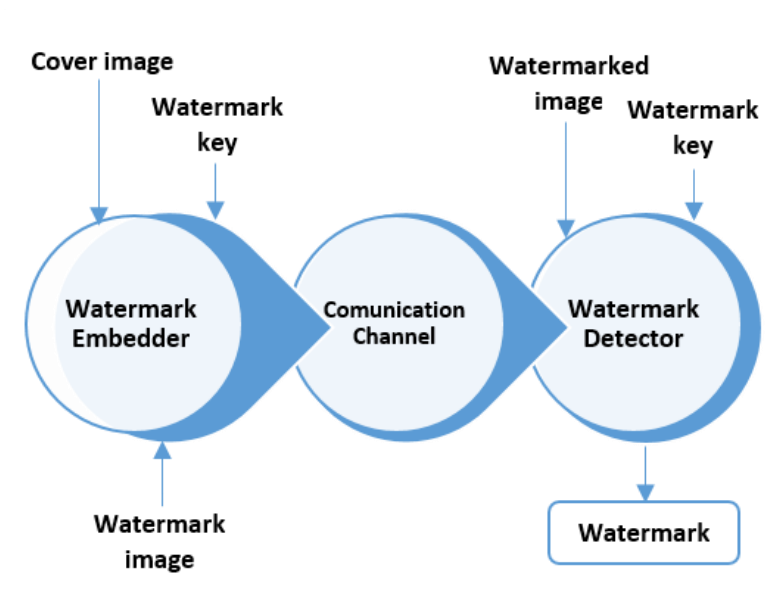

Fig 2.1: Basic Watermarking System

\section{PREVIOUS WORK}

In general, the embedding techniques can be categorized into two categories: spatial domain techniques or frequency domain techniques. Spatial domain watermarking techniques do a bit modifications on the pixels of one or two randomly selected subset of cover image. The general technique is LSB technique. Its modifications contain flipping the least significant bit of each pixel. The LSB technique is the simplest technique of watermark insertion. In still images, each pixel of the color image has three layers red, green and blue. Assuming that 3 bytes have been allocated for each pixel. Then, each color has 1 byte ( 8 bits), in which the intensity of that color can be specified on a range of 0 to 255 . For example, the value of a pixel is: $\mathrm{P} 0=\{\mathrm{R}=125, \mathrm{G}=245$, $B=50\}$. The value of another pixel is: $P 1=\{R=125, G=245$, 
$B=49\}$. Here all the value of $B$ have been changed. But how much of a difference does it make to the human eye? For the eye, detecting a difference of 1 on a color range of 256 is at all impossible. Now since each color is stored in a single byte, the last bit in each byte stores this difference of one. That is, the difference between values 225 and 224, or 150 and 149 is stored in the last bit, called the Least Significant Bit (LSB) [6]. Ever after this difference does not matter a lot, when we replace the color intensity information in the LSB with watermarking information, the image will still look the same to the naked eye [7]. Thus, for each pixel of 3 bytes ( 24 bits), 3 bits of watermarking information can be hidden, in the LSBs. This uses secret key to choose a random set of bits, and modify them with the watermark. Thus a simple algorithm for this technique would be:

Algorithm: LSB (Least Significant Bit) Watermarking

Input: Watermark W; Cover Image C; Secret Key.

Output: Watermarked Image WI.

Steps:

1. For random set of pixel using secret key in the image, $\mathrm{Pi}$

Do Loop: Store the next bit from $\mathrm{W}$ in the LSB location of $\mathrm{Pi}$ [red] byte, Store the next bit from W in the LSB position of Pi [green] byte, Store the next bit from $\mathrm{W}$ in the LSB position of $\mathrm{Pi}$ [blue] byte.

\section{End Loop.}

To extract watermark information, all the data in the LSBs of the color bytes need to be taken and be combined. Many frequency domains sometimes called the transform domains approaches have been proposed including the discrete cosine transform domain (DCT), discrete wavelet transform (DWT) and discrete Fourier transform (DFT). Frequency domain approach has some advantages because most of the operated signal processing can be well characterized in the frequency domain. In frequency domain, values of certain frequencies are modified from their original. The watermark is inserted into coefficients of the transformed image. These frequency modifications are done in the mid frequency components since the low frequency components are very responsive to distortion and the high frequency components can be deleted without so much affecting the original image quality. The frequency domain watermarking methods are comparatively robust to image processing, compression and noise compared with the spatial domain methods. Unfortunately, not too much data can be inserted in frequency domain because the quality of the host image will be distorted so much [8].

\section{PROPOSED METHOD}

In this section, the proposed method scheme (MSLDIP) will be presented which works on the spatial domain of the cover image. At the first the (SLDIP) will be presented before the (MSLDIP) method. SLDIP method takes the cover image and the watermark as input, convert the blue layer of the cover image into one row, and divide the row into blocks each of which contains 9 values, then consider the watermark is color image then each pixel will be represented in 3 bytes, according to the color image representation (which each pixel is specified by three values one each for red, blue, and green components of the pixel's color and each value represented by one byte, so each pixel will be represented in three bytes).
Each byte in the watermark image will be ranges from 0 to 255 , and make each byte value's length equal to 3 digits, for example we have byte of value 15 , this value equal to 015 which has length of 3 digits, finally substitute each 9 digits of each pixel with the last digit of each pixel in the current block, so each pixel of the watermark image will be embedded in only one block, and output the watermarked image and 2 keys which be required in the extraction process (Figure 4.1) and (Figure 4.2).

Assuming that watermark image of height 1 pixels and width 1 pixels, and cover image of height 3 pixels and width 3 pixels. The SLDIP will represent the cover image in one row which contains one block of 9 values $(125,255,086,192,145,210$, 035, 099, and 004), and the watermark image will be represented as $(230,105$, and 080$)$, the SLDIP will substitute 5 (last digit in cover image) in 125 with 2 (first digit in watermark image) in 230, the result is 122 and also substitutions done until reaching the last digit in the last value of the watermark. The watermarked image will be $(122,253$, 080, 195, 140, 215, 030, 098, and 000).

By using this method capacity of embedding has been increased, the maximum area of watermark image that can be embedded in any cover image can be caclulated by using this formula:

$$
\frac{\text { ImageWidth } \times \text { ImageHeight }}{9}=\text { watermark_area }
$$

If the watermark image is grayscale image this formula can be used:

$$
\frac{\text { ImageWidth } \times \text { ImageHeight }}{3}=\text { watermark_area }
$$

Supposing a (8 x 8) cover image, by using equation 1, we can embed colored watermark image of area 7 pixel $^{\mathbf{2}}$ which approximately equals to $(2 \times 3)$ colored watermark image, and by using equation 2 , a grayscale watermark image of area 21 pixel $^{2}$ which approximately equals to $(4 \times 5)$ grayscale watermark image can be embedded. Notice that SLDIP uses only one layer of the color image neither two nor three layers. This means that we can use this method in color and grayscale images [9].

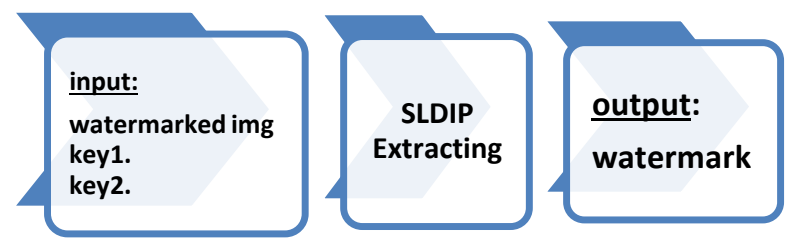

Fig 4.1: SLDIP Watermarking Embedding

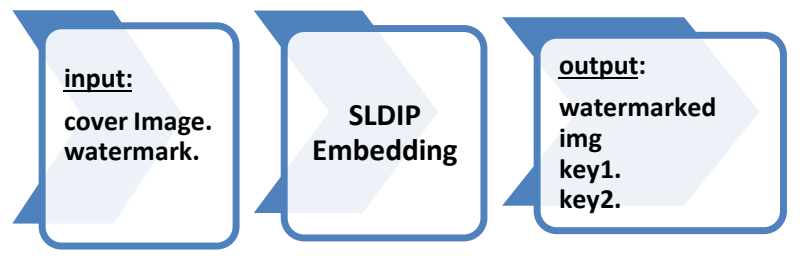

Fig 4.2: SLDIP Watermarking Extracting 
MSLDIP is a modification on SLDIP by update the substitution step to decrease the difference between the original pixel and the substituted pixel, for example embedding value digit 9 in pixel 100, by using SLDIP the pixel will be 109, but by MSLDIP two possible values can be taken for each substitution and choose the value that has the smallest difference, so the two values will be 109 and 99 , then the value with the smallest difference must be chosen, so the pixel value will be 99 , the difference will be 1 instead of 9 and this increases the PSNR value of the image.

\section{EXPERIMENTAL RESULTS}

In order to evaluate the performance of the watermarked images, there are some quality measures such as PSNR and MSE.

The MSE (Mean Square Root) is defined as an average squared difference between a reference image and a distorted image. It can be calculated by the formula given below

$$
M S E=\frac{1}{X Y}\left[\sum_{i=1}^{X} \sum_{j=1}^{Y}(c(i, j)-e(i, j))^{2}\right] \text { (3) }
$$

Where $\mathrm{X}$ and $\mathrm{Y}$ are height and width respectively of the cover image, the $c(i, j)$ is the pixel value of the cover image and $e(i, j)$ is the pixel value of the watermarked image.

The PSNR (Peak Signal to Noise Ratio) is an expression for the ratio between the maximum possible value (power) of a signal and the power of distorting noise that affects the quality of its representation. It can be calculated by the formula as

$$
P S N R=10 \log _{10}\left(\frac{L \times L}{M S E}\right)
$$

Where $\mathrm{L}$ is the peak signal value of the cover image which is equal to 255 for 8 bit images. [10].

SLDIP and MSLDIP have been implemented in MATLAB 2014 platform and the experiment has been conducted on various images (Figure 5.1).

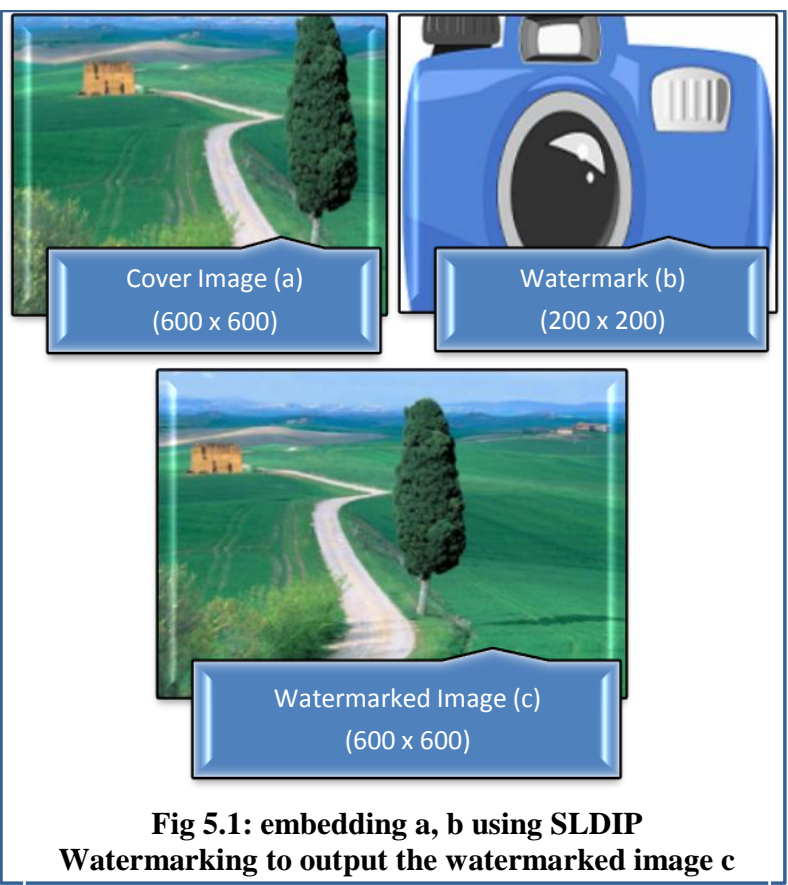

We SLDIP Watermarking has been applied on set of images different in sizes and calculate the Peak Signal to Noise Ratio (PSNR), and Mean Square Root (MSE), all results recorded in (Table 1).

Table 1. Results of applying SLDIP Watermarking on various sizes images

$\begin{array}{cccc}\begin{array}{c}\text { Cover } \\ \text { Image }\end{array} & \text { PSNR } & \text { MSE } & \begin{array}{c}\text { Watermark } \\ \text { Image }\end{array} \\ (600 \times 600) & 40.47 & 5.84 & (200 \times 200) \\ (768 \times 768) & 42.67 & 3.52 & (200 \times 200) \\ (1024 \times 1024) & 45.20 & 1.96 & (200 \times 200) \\ (1280 \times 1280) & 49.70 & 0.70 & (200 \times 200) \\ (1500 \times 1500) & 48.54 & 0.91 & (200 \times 200)\end{array}$

In (Figure 5.2), Chart showing the results of MSE and PSNR between the cover image and watermarked image in SLDIP.

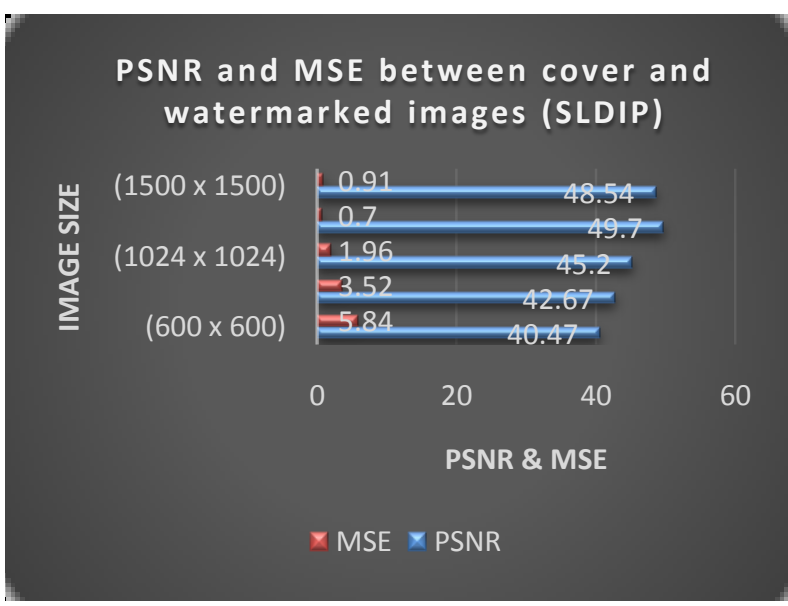

Fig 5.2: Chart showing the results of SLDIP

SLDIP has been compared with [11], SLDIP has been applied on Lena256 as cover image with watermark of size 48 byte color watermark image (384 bits) which greater than size of watermark in [11], however PSNR in SLDIP was $66.81 \mathrm{~dB}$, on the other side PSNR in [11] was $37.53 \mathrm{~dB}$.

Then MSLDIP Watermarking has been applied on the same set of images different in sizes and calculate the Peak Signal to Noise Ratio (PSNR), and Mean Square Root (MSE), all results recorded in Table (2).

Table 2. Results of applying MSLDIP Watermarking on various sizes images

\begin{tabular}{cccc}
$\begin{array}{c}\text { Cover } \\
\text { Image }\end{array}$ & PSNR & MSE & $\begin{array}{c}\text { Watermark } \\
\text { Image }\end{array}$ \\
\hline$(600 \times 600)$ & 40.47 & 5.84 & $(200 \times 200)$ \\
\hline$(768 \times 768)$ & 42.67 & 3.52 & $(200 \times 200)$ \\
\hline$(1024 \times 1024)$ & 45.20 & 1.96 & $(200 \times 200)$ \\
\hline$(1280 \times 1280)$ & 49.70 & 0.70 & $(200 \times 200)$ \\
\hline$(1500 \times 1500)$ & 48.54 & 0.91 & $(200 \times 200)$ \\
\hline
\end{tabular}


In (Figure 5.3), Chart showing the results of MSE and PSNR between the cover image and watermarked image in MSLDIP.

SLDIP and MSLDIP have been compared with [12], supposed four cover image with squared sizes $128,256,512$, and 1024 , and a watermark with full capacity with cover images according to [12], thus the full watermark capacity can be calculated using cover image sizes according to [12] by using formula

$$
\begin{aligned}
& \text { Round up }\left(\sqrt{\frac{\text { ImageWidth } \times \text { ImageHeight }}{24}}\right) \\
& =\text { watermark side length }
\end{aligned}
$$

Then values of PSNR and MSE of SLDIP are measured and compared with [12] in (Table 3) and values of PSNR and MSE of MSLDIP are measured and compared with [12] in (Table 4).

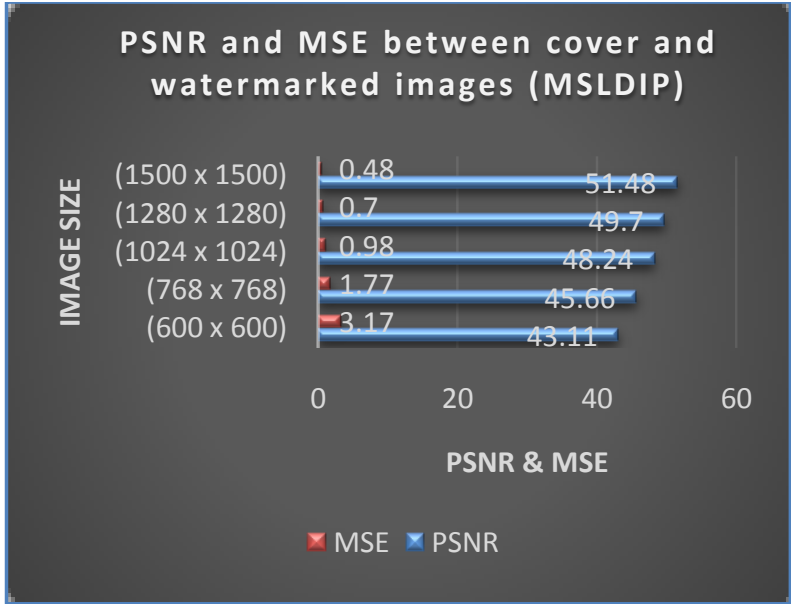

Fig 5.3: Chart showing the results of MSLDIP

Table 3. Results Comparison between [12] 4th Bit and SLDIP

\begin{tabular}{|c|c|c|c|c|c|}
\hline \multirow{2}{*}{ Cover Image } & \multirow{2}{*}{$\begin{array}{c}\text { Watermark } \\
\text { FC [12] }\end{array}$} & \multicolumn{2}{|c|}{ [12] 4th Bit } & \multicolumn{2}{|c|}{ SLDIP } \\
\cline { 3 - 6 } & PSNR & MSE & PSNR & MSE \\
\hline Baboon 128 & $(27 \times 27)$ & $31.68 \mathrm{~dB}$ & 44.50 & $44.49 \mathrm{~dB}$ & 2.32 \\
\hline Bird 256 & $(53 \times 53)$ & $31.68 \mathrm{~dB}$ & 44.50 & $44.74 \mathrm{~dB}$ & 2.18 \\
\hline Boat 512 & $(125 \times 125)$ & $31.68 \mathrm{~dB}$ & 44.50 & $43.05 \mathrm{~dB}$ & 3.22 \\
\hline
\end{tabular}

Table 4. Results Comparison between [12] $3^{\text {rd }}$ Bit and MSLDIP

\begin{tabular}{|c|c|c|c|c|c|}
\hline \multirow{2}{*}{ Cover Image } & Watermark & \multicolumn{2}{|c|}{ [12] 3th Bit } & \multicolumn{2}{|c|}{ MSLDIP } \\
\cline { 3 - 6 } & FC [12] & PSNR & MSE & PSNR \\
\hline Baboon 128 & $(27 \times 27)$ & $38.25 \mathrm{~dB}$ & 9.80 & $46.91 \mathrm{~dB}$ & 1.32 \\
\hline Bird 256 & $(53 \times 53)$ & $38.25 \mathrm{~dB}$ & 9.80 & $47.04 \mathrm{~dB}$ & 1.28 \\
\hline Boat 512 & $(125 \times 125)$ & $38.25 \mathrm{~dB}$ & 9.80 & $45.96 \mathrm{~dB}$ & 1.65 \\
\hline Pepper 1024 & $(210 \times 210)$ & $38.25 \mathrm{~dB}$ & 9.80 & $47.07 \mathrm{~dB}$ & 1.28 \\
\hline
\end{tabular}

From the comparison in table (3) and table (4), the reason of why SLDIP has been compared with [12] 4th Bit, and MSLDIP has been compared with [12] 3rd Bit? Has been clarified as, in SLDIP substitutions can change the value of pixel which the difference ranges from 0 to 10 and change in the 4th Bit in pixel can change the value of pixel which the difference ranges from 0 to 15 which include the SLDIP difference range, and in MSLDIP substitutions can change the value of pixel which the difference ranges from 0 to 5 and change in the $3 \mathrm{rd}$ Bit in pixel can change the value of pixel which the difference ranges from 0 to 7 which include the MSLDIP difference range. However results in SLDIP and MSLDIP are better.

After Implementing and analyzing the results, conclude that, the visual quality of the image doesn't change significantly, on the other hand this algorithm is more robust than LSB technique, because in LSB technique some attackers can possibly zero out several least significant bit of pixels of the image and hence clear the watermark. This technique has increased the capacity of watermark in embedding process.

\section{CONCLUSION}

Thus concluding that Digital Watermarking has importance in securing digital contents from unauthorized user. SLDIP and MSLDIP techniques have been implemented for this purpose, and from results conclude that the visual quality of the image doesn't change significantly, on the other hand this algorithm is more robust than LSB technique, because in LSB technique some attackers can possibly zero out several least significant bit of pixels of the image and hence clear the watermark. This technique has increased the capacity of embedding watermark. In the future more security will be considered to prevent unauthorized users from detecting the watermark from the image by using an encryption algorithm and more robustness will considered by using frequency domain. 


\section{REFERENCES}

[1] R.G. Schyndel, A. Tirkel, and C.F Osborne, "A Digital Watermark", Proceedings of IEEE International conference on Image Processing, ICIP, 1994.

[2] Christine I. Podilchuk, Edward J. Delp, "Digital watermarking: Algorithms and applications, IEEE Signal processing Magazine, July 2001.

[3] M. Abdullatif, A. M. Zeki, J. Chebil, and T. S. Gunawan, "Properties of Digital Image Watermarking".

[4] Preeti Gupta, "Cryptography based digital image watermarking algorithm to increase security of watermarked data", International Journal of Scientific \& Engineering Research, September 2012.

[5] Prabhishek Singh, R S Chadha, "A Survey of Digital Watermarking Techniques, Application and Attacks", IJEIT, March 2013.

[6] R. Chandramouli and Nasir Memon, "Analysis of LSB Based Image Steganography Techniques”, IEEE 2001.

[7] Muhammad Aamir Qureshi and Ran Tao, "A Comprehensive Analysis of Digital Watermarking", I.T. Journal: 471-475, 2006.
[8] Nisha Sharma, Neelam Malik, Kamlesh Sharma, "A Modified LSB Technique of Digital Watermarking in Spatial Domain", Manav Rahna International University, Aravali Hills Faridabad, March 2013.

[9] Ahmed A. Radwan, Ahmed Swilem, AlHussien Seddik, "A High Capacity SLDIP method", ICICIS, July 2011.

[10] Amit Kumar Singh, Nomit Sharma, Mayank Dave, Anand Mohan, "A Novel Technique for Digital Image Watermarking in Spatial Domain", 2012 2nd IEEE International Conference on Parallel, Distributed and Grid Computing.

[11] Chen Tao, Wang Jingchun, "Image Watermarking Method Using Integer to Integer Wavelet Transforms", TSINGHUA SCIENCE AND TECHNOLOGY, October 2002.

[12] Deepshikha Chopra, Preeti Gupta, Gaur Sanjay, Anil Gupta, "LSB based digital image watermarking for gray scale image", IOSRJCE October 2012. 